\title{
Two initially spherical bubbles rising in quiescent liquid
}

\author{
Manoj Kumar Tripathi ${ }^{\dagger}$, A. R. Premlata ${ }^{\dagger \dagger}$, Kirti Chandra Sahu ${ }^{\dagger \dagger}$, and Rama Govindarajan ${ }^{\dagger \dagger} *$ \\ ${ }^{\dagger}$ Indian Institute of Science Education and Research Bhopal 462 066, Madhya Pradesh, India \\ ${ }^{\dagger \dagger}$ Department of Chemical Engineering, Indian Institute of Technology Hyderabad, Sangareddy 502 285, Telangana, India \\ ${ }^{\dagger \dagger}$ International Centre for Theoretical Sciences, Tata Institute of Fundamental Research, Shivakote, Bengaluru 560089, India
}

A pair of bubbles starting from rest and rising side-by-side in a liquid have been shown earlier to display spherical and ellipsoidal shapes. In contrast to earlier computational studies on the two-dimensional dynamics of a pair of bubbles, we study the fully three-dimensional motion of the bubbles in the inertial regime. We reveal the destabilizing nature of the interaction between the wakes of the bubbles, which causes them to rise in an oscillatory path. Such three dimensionality sets in earlier in time than for a single bubble and also at a lower inertia. The interaction leads to a mirror symmetry in the trajectories of the two bubbles, which persists for some time even in the high inertia regime where each path is chaotic. The effect of the inertia and initial separation on the mirror symmetry of the path, the vortex shedding pattern and the attraction/repulsion between the bubbles are examined. The bubble rise has been interestingly observed to be symmetrical about the plane perpendicular to the separation vector for all separation distances considered in the present study.

\footnotetext{
* Email of the Corresponding Authors: ksahu@iith.ac.in, rama@icts.res.in
} 


\section{INTRODUCTION}

The phenomenon of gaseous bubbles rising together in liquid is not only encountered in many chemical and petrochemical applications, such as bubble column reactors, heat exchangers [1, 2], but also in natural phenomena [4]. In industrial applications, gaseous bubbles are frequently used to enhance the heat and mass transfer, and this enhancement depends primarily on the interaction and distribution of bubbles, apart from the flow characteristics. Thus the efficiency of these processes can be hugely influenced by topological changes in the bubbles, and the paths they follow. In this context, a fundamental understanding of the flow dynamics of simplified systems, such as the rising of a single bubble or two bubbles under the action of buoyancy can be very useful to analyse the above-mentioned complex flows involving many bubbles. Several researchers in the past have investigated the dynamics of rising of a single bubble or two bubbles in confined and unconfined media, which are briefly reviewed below.

The hydrodynamics of a single bubble in quiescent liquid has been studied both computationally (see e.g. [5-7]) and experimentally (see e.g. [8,9]). The experimental investigation on this subject provides a library of bubble shapes, including skirted, spherical cap, and oscillatory and non-oscillatory oblate ellipsoidal. Recently, Tripathi et al. [10] conducted three-dimensional numerical simulations of an initially spherical gaseous bubble rising under buoyancy in a liquid, and identified five different regions (shown in Fig. 1), which agree well with the gross features obtained in the experimental study of Bhaga \& Weber [9]. It was shown in the computational study [10] that in region I (which corresponds to low Eötvös number, Eo $\left(\equiv \rho_{o} g R^{2} / \sigma\right)$ and low Galilei number, $\left.G a\left(\equiv \rho_{o} \sqrt{g R} R / \mu_{o}\right)\right)$ the bubble maintains azimuthal symmetry. The bubble shapes in this region are either spherical, oblate or dimpled. In region II (high Eo and low $\mathrm{Ga}$ ), a bubble form axisymmetrical cap with a thin skirt trailing from the main body of the bubble (known as skirted bubble). A bubble in region III (low Eo and high $G a$ ) rises in a zigzag or a spiral path. A region III bubble remains its integrity but its shape changes with time due to the influence of relatively low surface tension force, but high inertial force as compared to an axisymmetric (region I) bubble. This phenomenon, commonly known as path instability [11-13]. A bubble in regions IV and V undergoes different types of break-ups, namely peripheral break-up (region IV) and central break-up (region V).

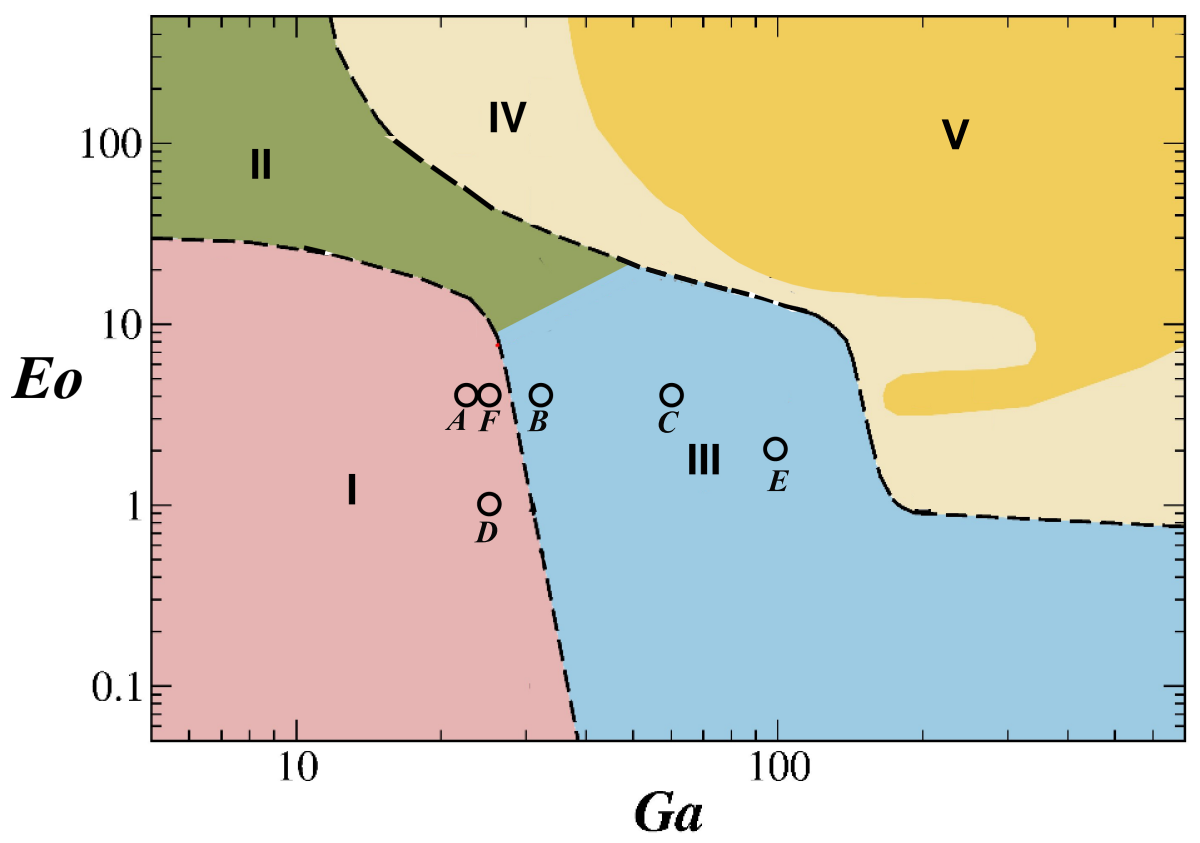

FIG. 1. Different regions in $G a-E o$ plane. Region I: axisymmetric; region II: skirted, region III: zigzagging or spiralling, region IV: peripheral break-up, and region V: central break-up. Also shown are the points $A, B, C, D, E$ and $F$ which corresponds to $(G a, E o)=(22.4,4),(32,4),(60,4),(25,1),(100,2)$ and $(25,4)$, respectively. These sets of $G a$ and Eo are considered in the present study. This figure is a modified plot taken from Tripathi et al. [10].

The dynamics of multiple bubbles have also been a subject of research for a long time. Many researchers, e.g. [14-16] investigated the interactions between flow and many bubbles in the context of bubble-column reactors, bioreactors, etc. However, in line with the context of the present study, we only review the previous investigations conducted on a pair of bubbles rising side by side. The hydrodynamics of two bubbles rising side-by-side not only get affected by direct interactions of the bubbles (which may result in coalescence for some parameter values), but also 
the interactions between the wakes or boundary layer developed around these bubbles, which can influence the shape deformations and paths of these bubbles. In the present study, we are mainly interested in the later phenomenon.

The interactions and trajectories of a pair of bubbles have been investigated analytically by Leal [17] in the Stokes flow and by a few researchers (see e.g. $[18,19]$ ) in the potential flow regimes. The analytical solution in the Stokes flow limit predicts the sedimentation of drops and interaction between slowly moving drops very well, whereas, the trajectories of bubbles have been predicted well in the potential flow limit by the solution proposed by Kok [18, 20]. He also experimentally showed that the two bubbles rising vertically in ultrapure water tend to rotate to align horizontally. By conducting two-dimensional simulations, Chen et al. [21] found that two bubbles rising side-by-side coalesce, and the resultant single bubble shows shape oscillations, which agrees with those observed in the experiment of Duinevald [22]. Duineveld [22] experimentally found that the zigzagging pair of bubbles have a higher amplitude of oscillation than a corresponding isolated bubble. He also noted that the potential theory fails to predict such motion because of the boundary layer interactions in the experiments. This has also been supported by the experiments and numerical simulations of Sanada et al. [23] and Legendre et al. [19], respectively. Sanada et al. [23] also experimentally investigated the bouncing and coalescence of a pair of bubbles rising side by side in an initially quiescent liquid. They found that for higher Reynolds numbers and low Weber numbers the bubbles approach each other and collide resulting either in coalescence or bouncing of the bubbles. They found that the Reynolds number at which the bubbles approach each other and collide decreases with an increase in the Morton number $\left(M o=E o^{3} / G a^{4}\right)$. However, they considered only less viscous surrounding fluid $\left(M o<1.14 \times 10^{-5}\right)$, and no information was given regarding the wake characteristics. Legendre et al. [19] predicted that for every Reynolds number there exists a separation distance for which the two effects, namely irrotational and wake effects due to viscosity, cancel each other and the drag of the two bubbles becomes equal to that of an isolated bubble. Also, it is correlated with the existence of a boundary in separation distance and the Reynolds number phase plot which separates the regions of attraction and repulsion between the bubbles. Recently, a few researchers [24, 26] also investigated rising of a pair of bubbles in shear-thinning fluids, and found that the attractive motion between the bubbles was increased with the increase in the shear-thinning tendency of the surrounding fluid.

As the above literature review shows, very few studies have been conducted to investigate the detailed threedimensional flow physics of deformable bubbles rising in a pair. Although there exist a few numerical studies (see for instance [27]) of two bubbles rising under buoyancy, their focus was mainly on validating the solver against existing experimental data. A detailed numerical study of a pair of non-deformable bubbles rising in a quiescent liquid was also conducted by Legendre et al. [19]. They used boundary fitted grids and considered only a quarter of the domain to simulate flow past fixed spherical bubbles. On the other hand, our study is fully three dimensional, which allows motion and deformation of bubbles in the three dimensional space. In contrast to Legendre et al. [19], we observe the zigzagging and spiralling motion of bubbles, whereas they always predicted a steady force acting between the bubbles. In the present work, we have investigated the rise of a pair of bubbles inside a quiescent liquid in the low inertial (high viscosity) regime and have compared it with the dynamics observed in case of single bubble.

The rest of the paper is organized as follows. The details of the problem formulation is provided in Section II. The results are discussed in Section III, and concluding remarks are given in Section IV.

\section{FORMULATION}

A schematic diagram of flow configuration considered in this study is given in Fig. 2. Two air bubbles (designated by ' 1 ' and ' 2 ' of fluid ' $i$ ') of equal radius $R$ rise side-by-side under the action of buoyancy inside a square channel of width $L$ and height $H$ filled with a liquid (designated by fluid ' $o$ '). A Cartesian coordinate system $(x, y, z)$ is used to model the flow dynamics. Gravity acts in the negative $z$ direction. At time $t=0$, the two spherical air bubbles ' 1 ' and ' 2 ' are kept at $(0,-q / 2,10 R)(0, q / 2,10 R)$, respectively. Initially, the air bubbles and the surrounding fluid are stationary. Three-dimensional numerical simulations are performed to understand the rising dynamics of the bubbles. The numerical method used is similar to the one used by Tripathi et al. [10], and is described briefly below.

In order to minimise the boundary effect, the outer boundaries are kept far away from the bubbles. Free-slip and no-penetration conditions are imposed on all the boundaries of the computational domain. The governing equations are the equations of mass and momentum conservation, given by

$$
\begin{aligned}
\nabla \cdot \mathbf{u} & =0, \\
\rho\left[\frac{\partial \mathbf{u}}{\partial t}+\mathbf{u} \cdot \nabla \mathbf{u}\right] & =-\nabla p+\nabla \cdot\left[\mu\left(\nabla \mathbf{u}+\nabla \mathbf{u}^{T}\right)\right]+\delta \sigma \kappa \mathbf{n}-\rho g \mathbf{j},
\end{aligned}
$$

where $\mathbf{u}=(u, v, w)$ denotes the velocity field, in which $u, v$ and $w$ represent the velocity components in the $x, y$ and $z$ directions, respectively. The interface separating the air and liquid phases is obtained by solving an advection 


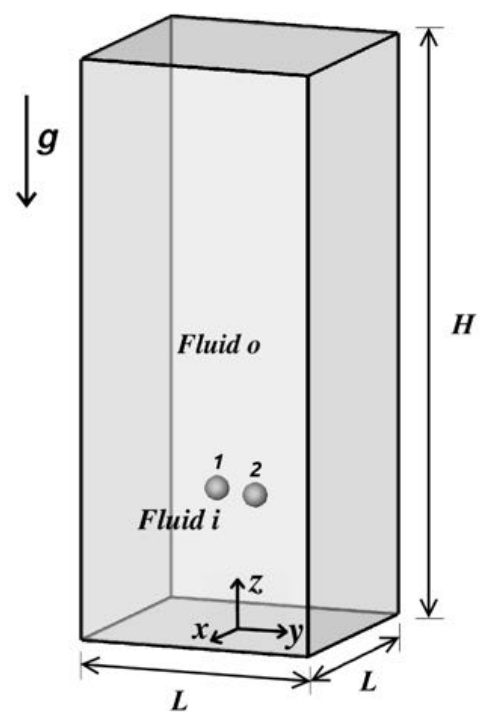

FIG. 2. Schematic diagram showing the initial configuration of the bubbles of equal radius $R$ (designated by ' 1 ' and '2') rising under the action of buoyancy inside a square computational domain of width $L$ and height $H$. The gaseous and the liquid phases are designated by ' $i$ ' and ' $o$ ', respectively. The two bubbles are initially separated by a distance $q$ along the $y$ coordinate, and placed at $z=10 R$ initially. The value of $L$, and $H$ are fixed at $40 R$ and $120 R$, respectively. The acceleration due to gravity, $g$ acts in the negative $z$ direction.

equation for the volume fraction of the liquid phase, $c(c=0$ and 1 for the air and liquid phases, respectively):

$$
\frac{\partial c}{\partial t}+\mathbf{u} \cdot \nabla c=0
$$

where $p$ is the pressure field, $t$ denotes time, $\mathbf{j}$ denotes the unit vector along the vertical direction, $g$ is the acceleration due to gravity, $\sigma$ represents the (constant) interfacial tension, $\delta$ is the Dirac delta function (given by $|\nabla c|$ ), and $\kappa=\nabla \cdot \mathbf{n}$ is the interfacial curvature, in which $\mathbf{n}$ is the outward-pointing unit normal to the interface.

The density, $\rho$, and the viscosity, $\mu$, are assumed to depend linearly on the liquid volume fraction $c$ as

$$
\begin{aligned}
& \rho=c \rho_{o}+(1-c) \rho_{i}, \\
& \mu=c \mu_{o}+(1-c) \mu_{i},
\end{aligned}
$$

where $\rho_{i}, \mu_{i}$ and $\rho_{o}, \mu_{o}$ are the density and dynamic viscosity of the air and the liquid phases, respectively.

The following scaling is used to non-dimensionalise the above governing equations:

$$
[x, y, z, q]=R[\widetilde{x}, \widetilde{y}, \widetilde{z}, \widetilde{q}], t=\frac{R}{V} \widetilde{t}, \mathbf{u}=V \tilde{\mathbf{u}}, p=\rho_{o} V^{2} \widetilde{p}, \mu=\mu_{o} \widetilde{\mu}, \rho=\rho_{o} \widetilde{\rho}, \delta=\widetilde{\delta} / R,
$$

where the velocity scale is $V=\sqrt{g R}$, and the tildes designate dimensionless quantities. After dropping tildes from all nondimensional variables, the governing dimensionless equations are given by

$$
\begin{aligned}
\nabla \cdot \mathbf{u} & =0, \\
\frac{\partial \mathbf{u}}{\partial t}+\mathbf{u} \cdot \nabla \mathbf{u} & =-\nabla p+\frac{1}{G a} \nabla \cdot\left[\mu\left(\nabla \mathbf{u}+\nabla \mathbf{u}^{T}\right)\right]+\delta \frac{\nabla \cdot \mathbf{n}}{E o} \mathbf{n}-\rho \mathbf{j}, \\
\frac{\partial c}{\partial t}+\mathbf{u} \cdot \nabla c & =0,
\end{aligned}
$$

where the dimensionless density and dynamic viscosity are given by

$$
\begin{aligned}
& \rho=c+(1-c) \rho_{r}, \\
& \mu=c+(1-c) \mu_{r} .
\end{aligned}
$$

Here $\rho_{r}\left(\equiv \rho_{i} / \rho_{o}\right)$ and $\mu_{r}\left(\equiv \mu_{i} / \mu_{o}\right)$ are density and viscosity ratios, respectively. An open-source fluid flow solver, Gerris created by Popinet [28] is used in the present study. The present numerical method uses the framework of 
a Volume-of-Fluid (VOF) approach that incorporates the surface tension term as a body force in the Navier-Stokes equations [29]. In order to minimise the problem of spurious currents, which one encounters while dealing with large density and viscosity ratios, a height-function based balanced-force continuum-surface-force formulation is used [30]. In order to ensure the accuracy of the results, a dynamic adaptive grid refinement is incorporated based on the vorticity magnitude and bubble interface. This solver was also validated extensively by comparing with the previous numerical and experimental results (see Tripathi et al. [10]). Figure 15 (see appendix) also shows a comparison of the terminal shapes of an air bubble rising in a liquid obtained from the present solver with those of Bhaga \& Weber [9] for different values of Galilee number. The results obtained from the present study are discussed next.

\section{RESULTS}

The non-dimensional parameters in this problem are the Galilee number, the Eötvös number and the initial horizontal separation $q$ between the bubbles, expressed as a multiple of a bubble radius. The first two, as mentioned above, are defined respectively by

$$
G a \equiv \frac{\rho_{o} g^{1 / 2} R^{3 / 2}}{\mu_{o}}, \quad \text { and } \quad E o \equiv \frac{\rho_{o} g R^{2}}{\sigma} .
$$

$G a$ is a ratio of the inertial to the viscous forces in the problem. It is effectively a Reynolds number, with the velocity scale based on gravity $(\sqrt{g R})$. Eo is also known as the Bond number, giving the ratio of gravitational to surface forces. Throughout this study, the values of the density and viscosity ratios are kept fixed at $10^{-3}$ and $10^{-2}$, respectively. First, we study the effect of varying the Galilei number and the initial separation in turn, for a fixed Eo $=4$.

\section{A. Effect of bubble inertia $(G a)$}

The dynamics of two bubbles rising side-by-side are shown for different inertia, i.e., for $G a=22.4,32$ and 60 in Fig. $3(\mathrm{a})$, (b) and (c), respectively. The Eötvös number is kept fixed at $E o=4$, and initially the bubbles are separated by a distance $q=3$ in the $y$ coordinate. These values of $G a$ are chosen because they represent qualitatively distinct regimes of the behaviour of a single bubble rising in an initially quiescent liquid. This can be seen in Fig. 1, where these parameters are denoted by small circles termed as $A, B$ and $C$. In the context of a single bubble, point $A$ lies well within region I, where the bubble would adopt an axisymmetric shape and, after some initial transience, attain a terminal velocity. Point $C$ lies in region III, where a single bubble would execute zigzagging/spiralling motion. Point $B$ lies in the latter region, but very close to the border between regions I and III. We now wish to examine how the behaviour of the bubble gets modified by the presence of another bubble of the same size rising simultaneously. The horizontal location of the centre of gravity of each bubble is denoted by $y_{C G}$ and $x_{C G}$ in the $y$ and $x$ coordinates, respectively. These positions of the center-of-gravity of the bubbles are presented in the top and bottom panels of Fig. 3(a), (b) and (c), respectively as the bubbles rise, as functions of the height $z$. The path of bubble ' 2 ' has been mirrored about the $x$ axis, so that one may easily examine whether the dynamics is symmetric. In order to compare the rise dynamics with the single bubble behaviour, the variations of $y_{C G}$ and $x_{C G}$ obtained for a single bubble rising in the same liquid are plotted by black dot-dashed lines.

The effect that is immediately evident is that the bubbles move away from each other progressively, i.e., their horizontal separation in $y$ shows an overall tendency to increase. This increase is modulated by oscillations. At low inertia, where a single bubble would merely have travelled vertically upwards at constant velocity, the two bubbles show small amplitude oscillations in the $y$ - $z$ plane as they move away from each other (Fig. 3(a)), and their motion in the $x$-axis is negligible, so the dynamics remains two-dimensional. For $G a=32$ and 60 (Fig. 3(b,c)), the frequency of pitch of the spiralling motion of the bubbles in the $y-z$ plane is 0.12 . In the case of single bubble, as well, it is nearly the same (0.118). For $G a=22.4$, the two bubbles oscillate at a frequency 0.11 (Fig. 3(a)), but the single bubble does not oscillates but merely migrates vertically along the axis of symmetry. The bubbles for $G a=22.4$ migrate away from each other achieving a terminal vertical velocity and a small spreading angle (made with the $z$ axis) of radians. This migration is modulated by small amplitude oscillations. Thus the transition to oscillatory behaviour sets in at a lower Galilee number than in the single bubble case. In other words, the dynamics of a single bubble for $G a=22.4$ and $E o=4$ lies completely in the axisymmetric region (region I in Fig. 1), i.e., the bubble rises along a vertical line, whereas the two bubbles display an increasing separation modulated by small amplitude oscillations. The bubbles for $G a=32$ also move away from each other at a spreading angle (with the $z$ axis) of 0.017 radians, but modulated now by high amplitude oscillations. In contrast, the dynamics of the bubbles for $G a=60$ appears to be chaotic. For two bubbles, the amplitude of oscillations at later times are $0.175,0.708$ and 1.2 for $G a=22.4,32$ and 60 , respectively. 
(a)

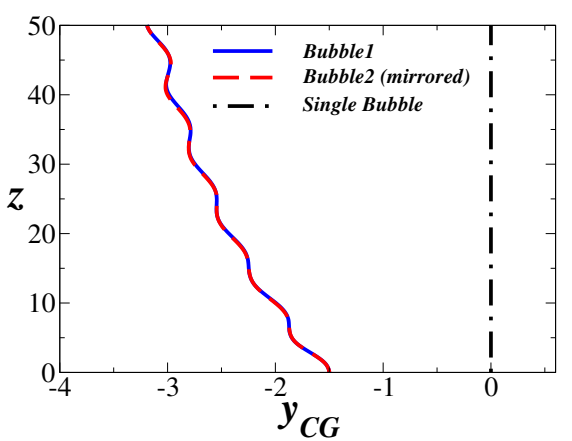

(d)

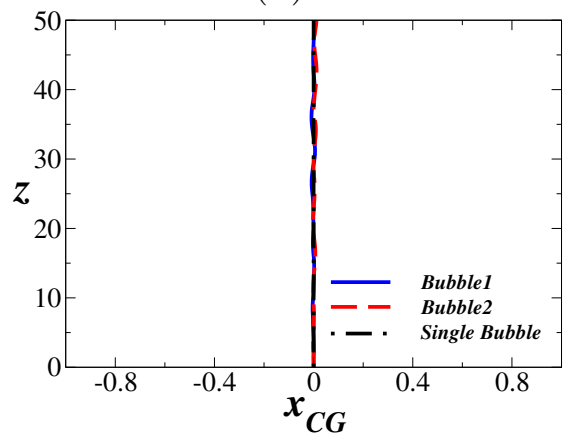

(b)

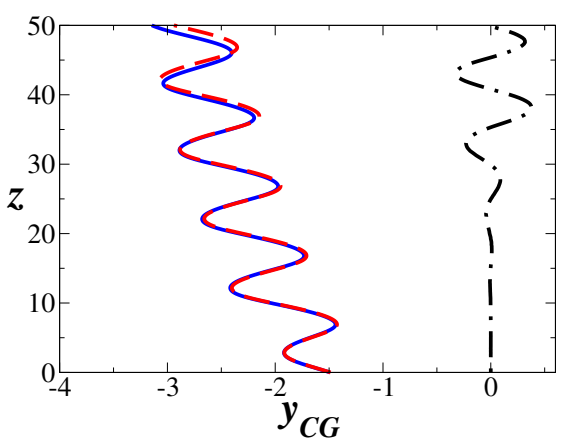

(e)

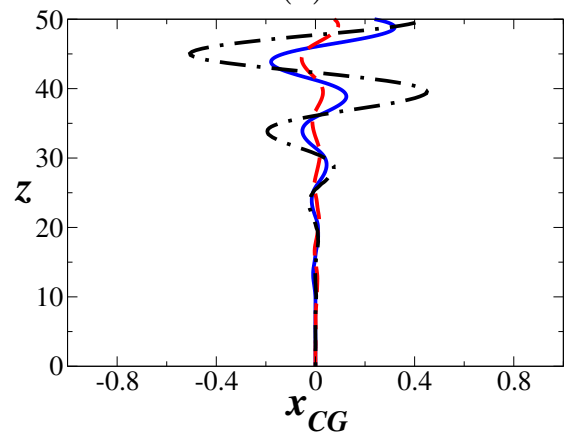

(c)

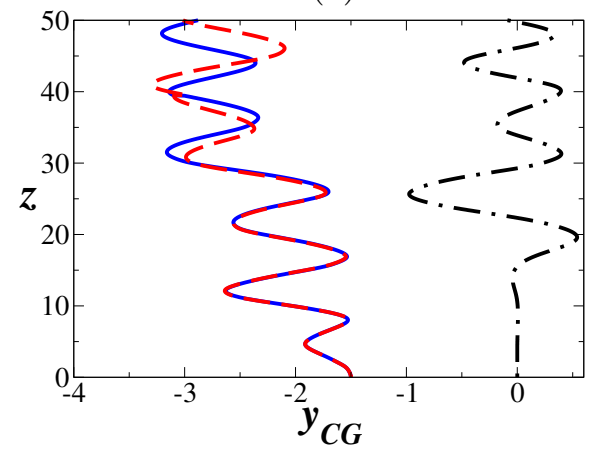

(f)

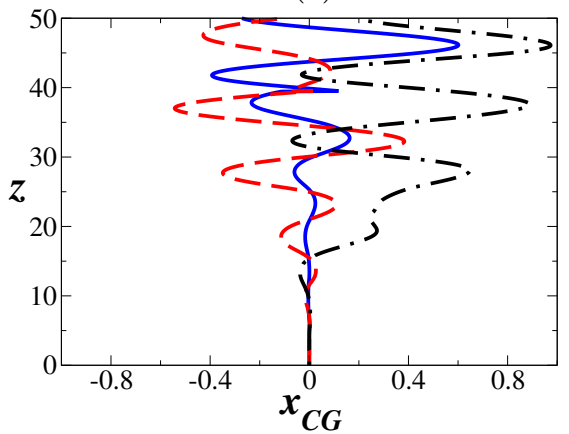

FIG. 3. Variation of $y_{C G}$ (top panels) and $x_{C G}$ (bottom panels) along the vertical path of the two bubbles released side by side. The Galilei numbers in each case are (a,d) $G a=22.4,(\mathrm{~b}, \mathrm{e}) G a=32$, and (c,f) $G a=60$. The rest of the parameter values are $E o=4, q=3, \rho_{r}=10^{-3}$ and $\mu_{r}=10^{-2}$. The position of the bubble initially placed at positive $y$ (bubble '2') has been mirrored about the $x$ axis in order to compare its path with that of the other bubble. The black dot-dashed lines represent the path a single bubble released at $(x, y, z)=(0,0,10 R)$ would follow.

(a)

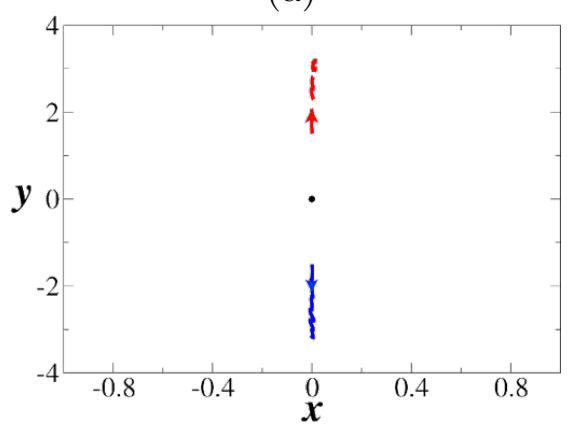

(b)

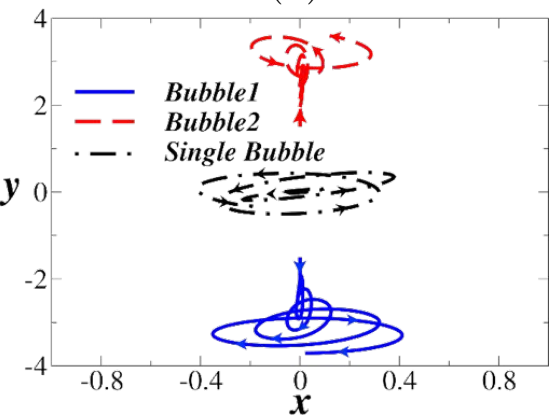

(c)

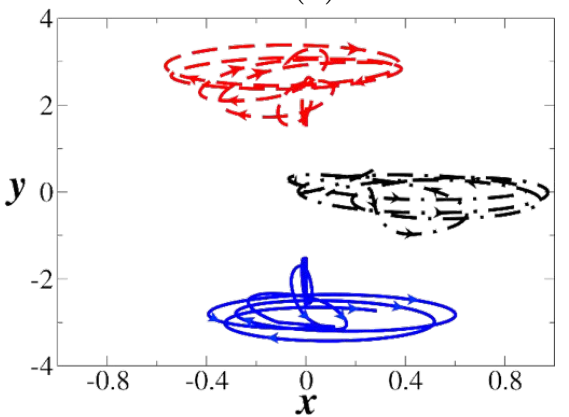

FIG. 4. The top view of the trajectories of the two bubbles for (a) $G a=22.4$, (b) $G a=32$, and (c) $G a=60$. The top view of the trajectories of the single bubble are also shown by black dot-dashed lines. The other parameter values are the same as in Fig. 3.

In the case of a single bubble, the amplitude of oscillations are much smaller, at 0.0317 and 0.157 for $G a=32$ and 60 , respectively. Inspection also reveals that for $G a=32$ and 60 , single bubble rises in a straight vertical path initially and then oscillates later. However, it can be seen that the oscillations start from $t=0$ in case of two bubbles rising simultaneously. Thus the presence of the second bubble significantly increases the amplitude of oscillations, decreases the time of onset of oscillations as well as decreases the $G a$ at which the transition from steady to oscillatory motion occurs. These changes due to the presence of the second bubble can be directly correlated with the changes in vortex shedding, as will be seen later. In the case shown, the paths of the two bubbles are perfect mirror images of each other about the $x$-axis. When we examine the top view shown in Fig. 4(a), the systematic increase in separation between the bubbles is apparent, and it is clear that the motion is confined to the $y-z$ plane.

At a higher Galilei number (Fig. 3(b)) a single bubble begins to display spiralling motion. The two bubbles execute 


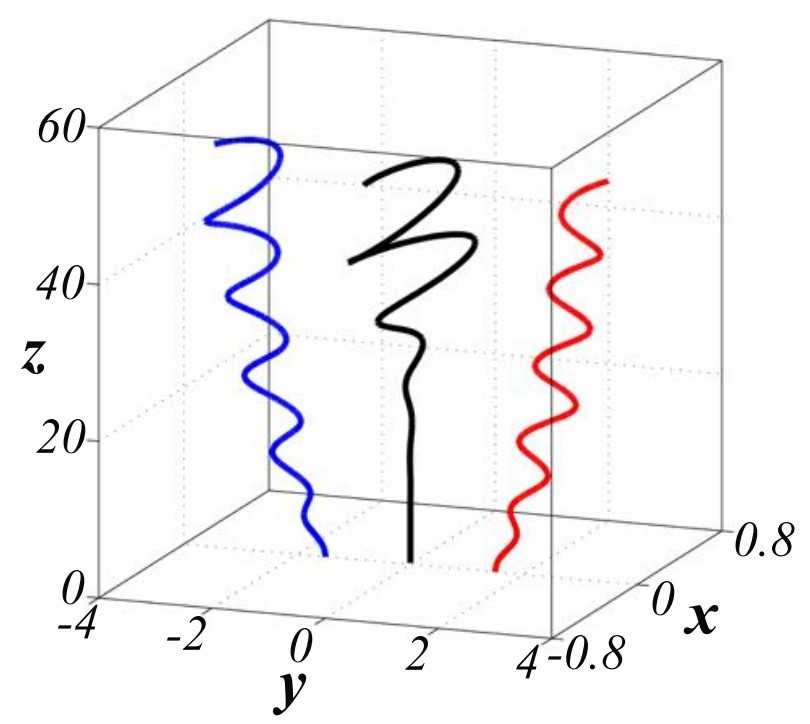

FIG. 5. Three dimensional trajectories of single bubble (shown in black) and bubble pair (shown in indigo and red) for $G a=32$. The rest of the parameter values are the same as in Fig. 3.

spiralling motion too, while each increases its distance from the other. A breaking of mirror-symmetry is already in evidence. Note that the scale for $x_{C G}$ is exaggerated compared to the one for $y_{C G}$, but it is clear that the motion is three dimensional now. The top view in Fig. 4(b) shows how an initial rapid repulsion is followed by a slowly widening spiral motion. For purposes of illustration, the three dimensional trajectories in this case are shown in Fig. 5. Superimposed on the moving apart of the two bubbles, a spiralling motion is seen whose pitch is comparable to its radius, and whose amplitude at later times is smaller than that of a single bubble. At an even higher Galilei number (Fig. 3(c) and Fig. 4(c)) the dynamics is fully three dimensional, and the asymmetry in the tracks of the two bubbles is very noticeable, as is the irregularity in the spiralling motion. Oscillations set in sooner for the two bubbles case than for a single bubble.

The trajectories we have seen lead us to expect oscillations in the horizontal velocity components as well, and these are shown as functions of time for the three Galilei numbers in Fig. 6 (a) and (b). The temporal variations of vertical velocity component of the bubble, $v_{b z}$ are also shown in Fig. 6 (c) for different values of $G a$. Interestingly, for the higher Galilei numbers, the vertical velocity shows oscillations (Fig. 6 (c)) as well, with the bubbles alternatingly rising slower and faster. The frequency of this variation is twice than that of the horizontal components. The in-plane velocities in the phase portrait of Fig. 6 (d) are therefore seen to follow figures of 'eight'. This oscillation in the vertical velocity is caused by the breaking of left-right symmetry in the two-bubble configuration, and is absent in the single bubble velocity pattern. It can also be seen in Fig. $6(\mathrm{~b}, \mathrm{c})$ that only for low Galilei number $(G a=22.4)$, the left bubble achieves a terminal velocity in the $z$-direction $\left(v_{b z}\right.$ is approximately equals to 1.05), and the variations in other components of bubble velocity, $v_{b x}$ and $v_{b y}$ are very small. The average values of $v_{b x}$ and $v_{b y}$ for $G a=22.4$ are about 0 and -0.02 , respectively. The motions of the right and left bubbles are symmetrical about the $x$ axis. Thus, the bubbles for low $G a$ move away from each other very slowly in the $y-z$ plane at $x=0$. In other words, for $G a=22.4$ and $E o=4$, a single bubble rises along a vertical line, whereas the two bubbles display an increasing separation modulated by small amplitude oscillations. The dynamics of the bubbles at high $G a$ values is three dimensional. The transition from two-dimensional motion to three-dimensional motion occurs at $G a \approx 30$ for $E o=4$. The mechanism of this transition was studied by Cano-Lozano et al. $[31,32]$ via a stability analysis. Their study consisted of perturbations on an axisymmetric bubble shape, whereas the presence of another bubbles breaks the axisymmetry of the flow around a given bubble at all $G a$. We have seen that this promotes three-dimensionality. For $G a=60$, the motion appears to be chaotic. The dimensionless force of attraction and repulsion acting on bubble ' 1 ' and ' 2 ', which are defined as $m_{b} a_{1}$ and $m_{b} a_{2}$, are plotted in Fig. 7. Here, $m_{b}$ represents the mass of each bubble, and $a_{1}$ and $a_{2}$ represent the accelerations of bubble ' 1 ' and '2' in the $y$ direction, respectively. Kok [18] derived the equations of motion for a spherical bubble in the Lagrangian framework using a potential flow approximation, which was later used by De Vries et al. [25] after taking into account the dipole interactions between a vertical wall and a bubble. Additional physics was accounted for in these equations by the authors to obtain the repeated bouncing, sliding and lift effects in the model. The dimensionless lift force derived by De Vries et al. [25] for their experiments is given by $L \approx \pi U_{T}^{2} / 13$, 
(a)

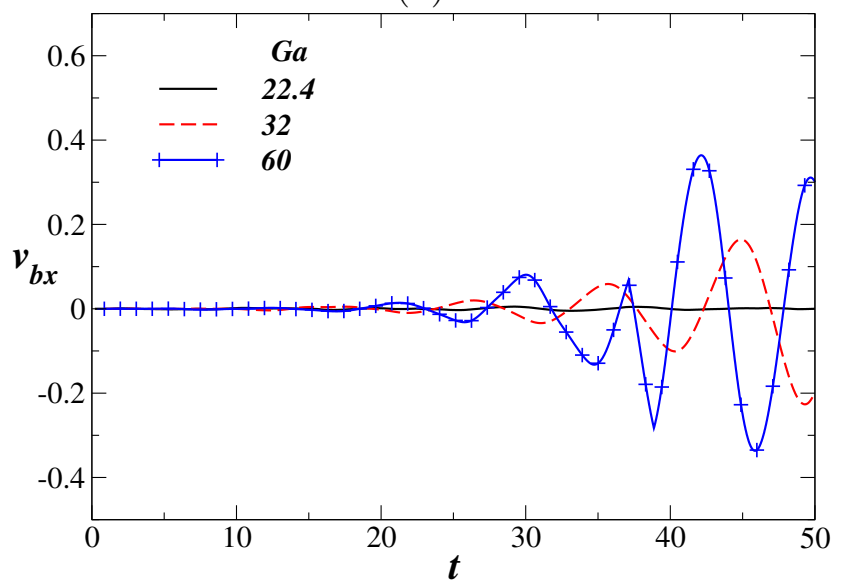

(c)

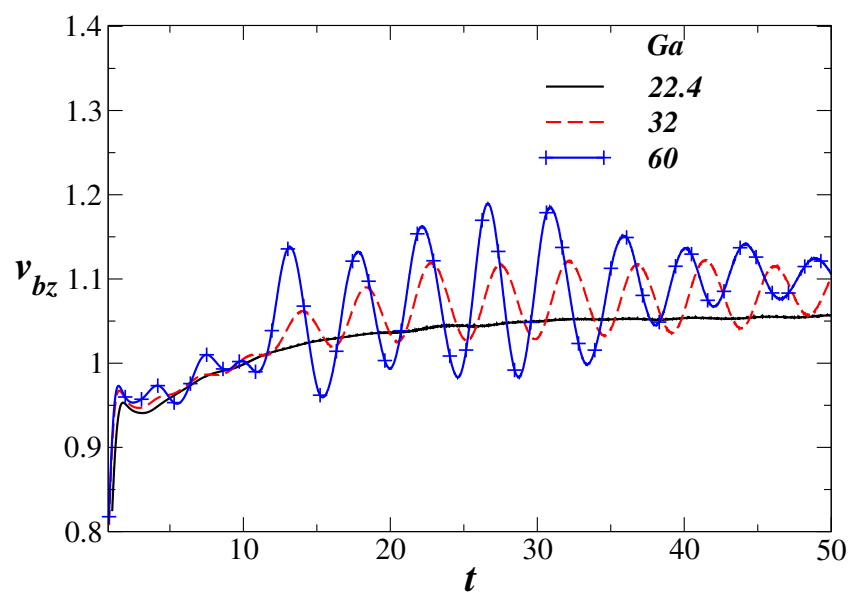

(b)

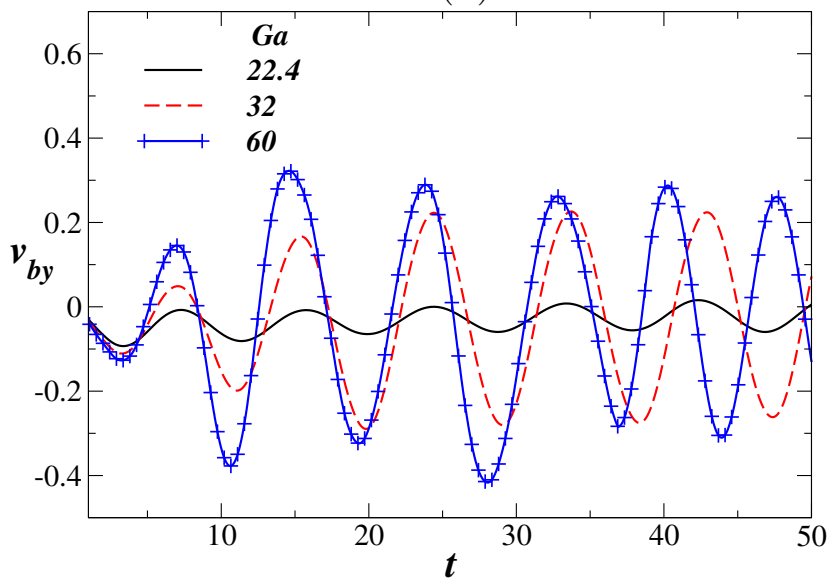

(d)

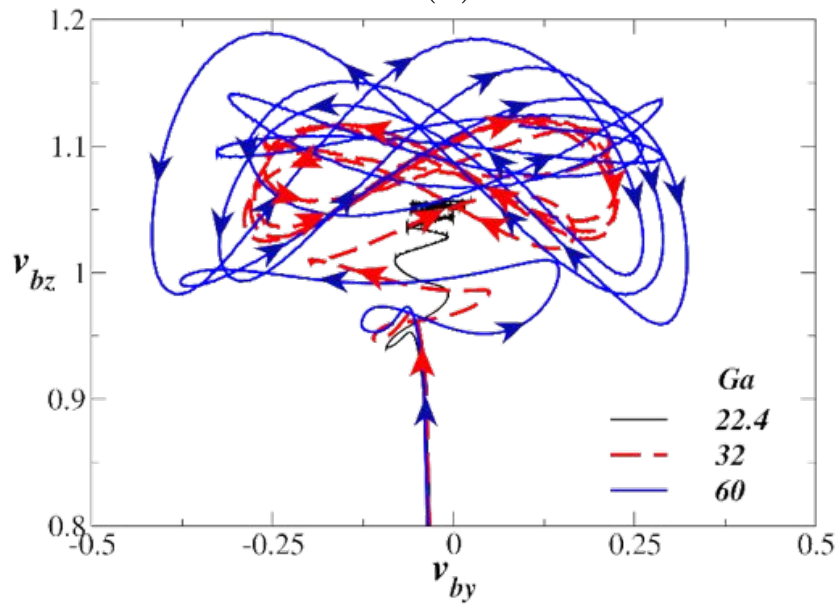

FIG. 6. Temporal variations of the velocity, $v_{b}$ of the left bubble (bubble ' 1 ') for different values of $G a$ in the (a) $x$, (b) $y$ and (c) $z$ directions. The components $v_{b}$ in the $x, y$ and $z$ directions are $v_{b x}, v_{b y}$ and $v_{b z}$, respectively. (d) Phase portrait ( $v_{b y}$ versus $v_{b z}$ plot). The other parameter values are the same as in Fig. 3.

which in dimensionless form is 0.24 . Our bubbles are not restricted to a plane, and are seen to undergo spiralling motion. The main force of one bubble on the other is thus in the azimuthal (horizontal component along the spiral) rather than the radial direction $(y)$. Accordingly the lift forces obtained in the present study (Fig. 7) are two orders of magnitude smaller as compared to the magnitudes suggested by these authors. Also, the lift forces obtained by Hallez \& Legendre [3] were of the order of 0.05 for $G a \approx 100$ and $q=1$, which too is an order of magnitude larger than the results of the present study. It is to be noted that in the case of Kok [18], De Vries et al. [25], and Hallez $\&$ Legendre [3] the bubbles were spherical with no motion in the $x$-direction. Our study displays the importance of a three-dimensional study of bubbles in the flow regime considered in our investigation.

In order to understand the associated shape deformation, while the two bubbles are moving away (for $G a=22.4$ ) or spiralling (for $G a=60$ ), the spatio-temporal evolutions of shape of bubbles are shown for these Galilei numbers in Figs. 8 and 9, respectively. Two views: tilted-side and top views are shown. The shape evolutions of the corresponding case of rising of single bubble are also shown in the right panel of each figure. It can be seen in Fig. 8(a) that the two bubbles deform to steady oblate shapes, which look similar to that observed for the single-bubble case. The bubbles remain circular when viewed from the top (Fig. 8(b)) as they move away from each other. Unlike for $G a=22.4$, in the high Galilei number case $(G a=60)$, it can be seen in Fig. 9(a) and (b) that the bubbles undergo unsteady large asymmetrical deformations, which are evident in both the tilted-side and top views. The deformations are similar to those displayed by a single bubble, but set in sooner than for a single bubble, this difference is visible at $t=10$ in the figure.

The effect of one bubble on the other is most apparent in the vortex shedding patterns, as shown for different Galilei numbers in Fig. 10(a,b,c) at $t=15$ and (d,e,f) at $t=30$. The positive and negative values are shown by red and green colors respectively for $\omega_{z}= \pm 0.3$. At the low Galilei number $(G a=22.4$; see Fig. 10(a,d)) of regime I 
(a)

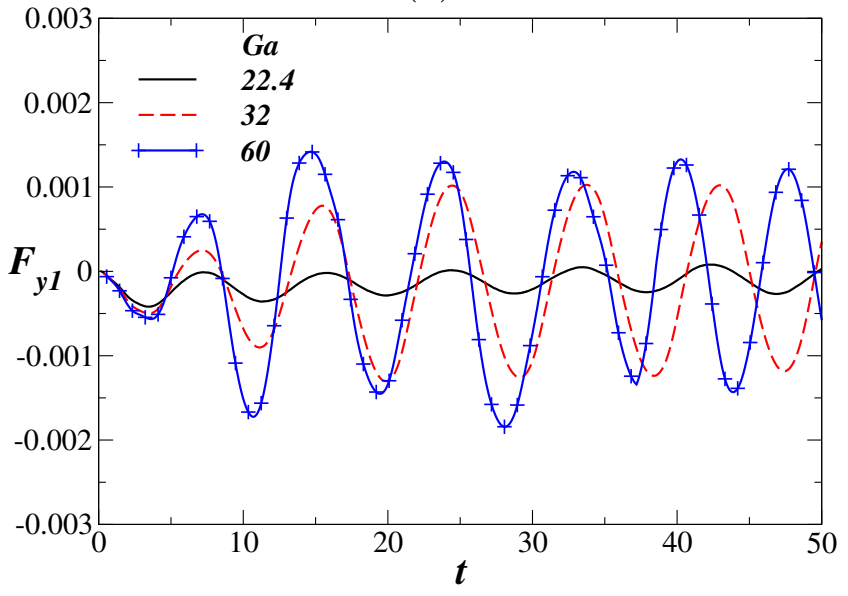

(b)

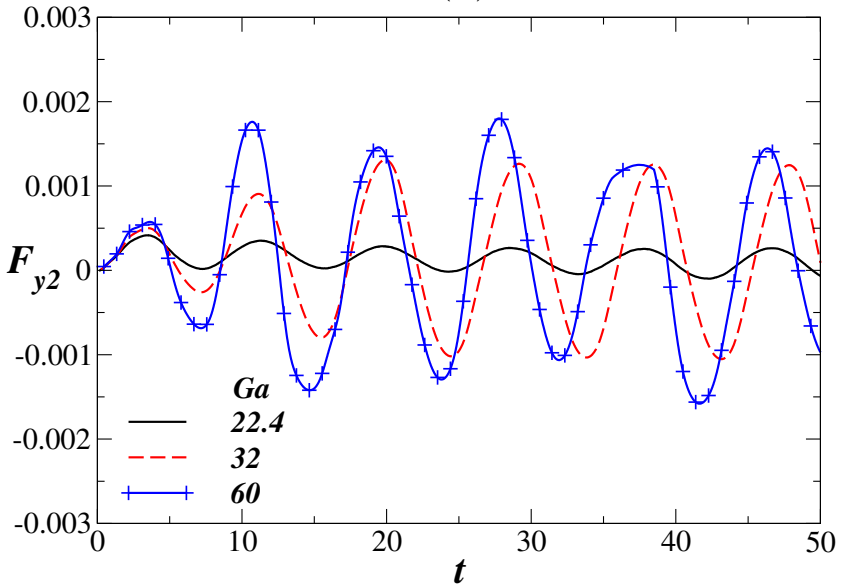

FIG. 7. Temporal variations of lateral force (in the $y$ direction) acting on bubble ' 1 ' and '2' for different values of $G a$. The other parameter values are the same as in Fig. 3.

(a)

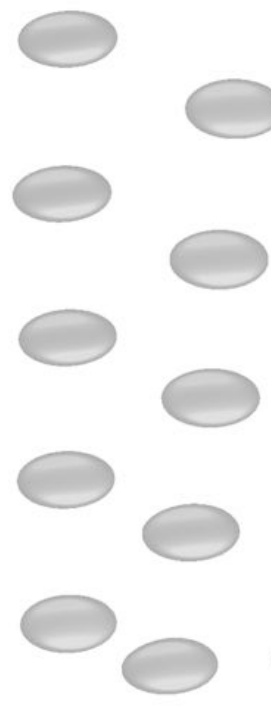

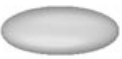
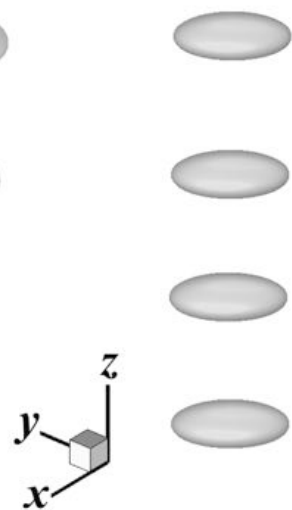

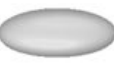

(b)
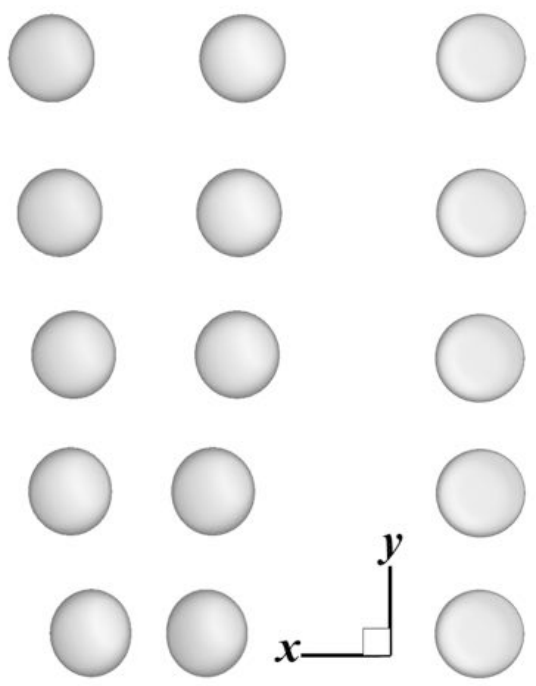

FIG. 8. Spatio-temporal variation of the shape of the bubble in (a) tilted-side view, and (b) top view for $G a=22.4$. From bottom to top: $t=10,20,30,40$ and 50. The remaining parameter values are the same as in Fig. 3 .

motion, there is no visible vorticity in the case of a single bubble, but vorticity is developed in the equatorial plane when two bubbles rise side-by-side. Further the wake of each is distorted from a straight path by the presence of the other wake, with the oppositely signed vortices being drawn to each other. This lead to an asymmetry in the pressure distribution on each bubble, resulting in repulsive motion rather than a solely vertical rise. With the increase in the Galilei number (for $G a=32$, see Fig. 10(b,e)), vortex shedding occurs in the wake regions in case of two bubbles. It is to be noted that when we plot the vorticity contours for $\omega_{z}= \pm 0.05$ or less, we observe vortex shedding even in case of single bubble at $G a=32$, but vortex shedding does not appear for both the single and two bubbles at $G a=22.4$. This is a strong evidence that vortex shedding is the mechanism behind the oscillatory motion of the bubbles. The vortex shedding becomes more intense for high Galilei number $(G a=60)$ as shown in Fig. 10(c,f). For $G a=60$, a smaller tail (less intense) of vortex shedding appears for the single-bubble case. For the intermediate and high Galilei numbers, the shed vortex from each bubble alternates in sign with time. This results in oscillatory and spiralling behaviour. The shedding from one bubble moreover is out of phase with the shedding from the other bubble and again there is a distortion in the shed pattern, resulting in overall increase in bubble separation, as was 
(a)

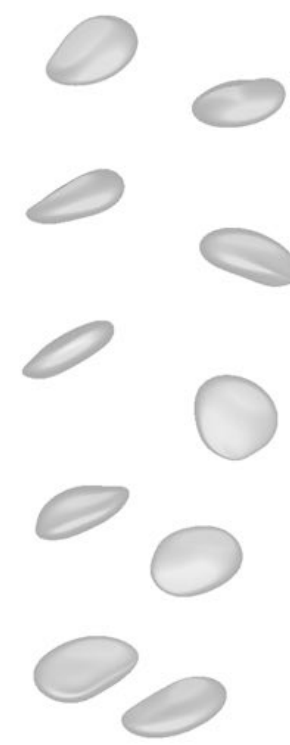

(b)

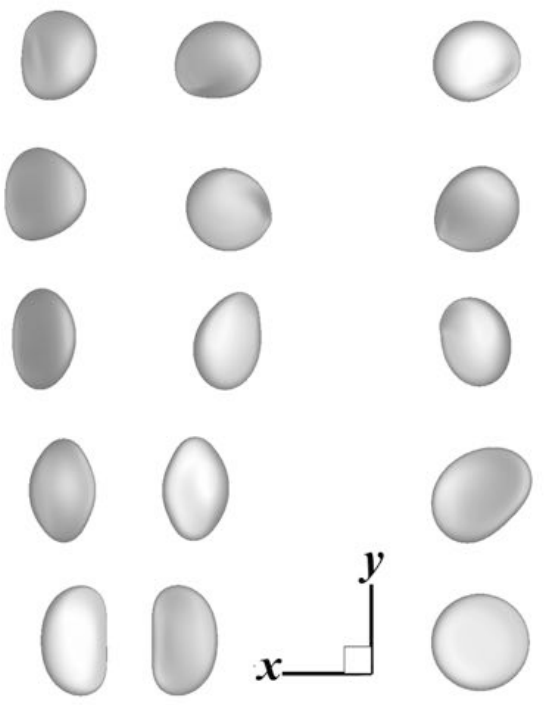

FIG. 9. Spatio-temporal evaluations of shape of the bubble for (a) tilted-side, (b) top view for $G a=60$. From bottom to top: $t=10,20,30,40$ and 50. The rest of the parameter values are the same as in Fig. 3.

seen in figure 3.

\section{B. Effect of initial separation, $q$}

We now examine the effect of initial separation between the bubbles, for a fixed Galilei number of 32 . The trajectories are shown in the $y-z$ and $x-z$ planes in figure 11. Two features are noteworthy. The first is that the initial increase in bubble separation is stronger when the bubbles are nearer to each other, while at later times they settle into a trajectory which, in the $y-z$ plane alone, does not depend on the initial separation. The frequencies of oscillations for $q=2.2,3$ and 4 are found to be $0.1,0.12$ and 0.12 , respectively. The amplitude and frequency of oscillations appear to be insensitive to $q$ for the parameter values considered in this study. The speed of the bubbles along the line of separation is shown in figure 12, and it is seen that barring a phase, all three initial separations settle into the same oscillation in the speed along the $y$ direction. Thus, for initial separations below a threshold, the dynamics, in the $y-z$ plane alone, at this Galilei number follows a common pattern except at early times.

The second striking feature of this dynamics is that the three-dimensional nature of the trajectories is highly dependent on the initial separation. Bubbles which start life close to each other, remain tightly bound in their futures, displaying trajectories that are mere mirror images of each other, as seen in figure 11(a), top and bottom panels. However, this symmetry is broken when bubbles start life further apart. One of the bubbles oscillates gently into the third dimension whereas the other displays large forays in the $x$ (cross) direction. While the dynamics remains out-of-phase in the $y-z$ plane, the phase in the $x-z$ plane of one bubble is not tied to the other. The resulting complete trajectories are seen in figure 13. For $q \geq 3$, one of the bubbles has a significantly lower spiralling radius than the other. At all initial separations, both bubbles have smaller spiralling amplitudes than a single bubble does under the same conditions.

\section{Some other cases}

In order to understand how the dynamics changes if we slightly vary the parameters, we investigate motion of bubbles for some other sets of $(G a, E o)$, designated by points $D(G a=25, E o=1), E(G a=100, E o=2)$ and $F(G a=25, E o=4)$ in Fig. 1. In Fig. 14(a), the variations of $y_{C G}$ and $x_{C G}$ in the $z$ directions are plotted for bubble ' 1 ' and bubble '2' (mirrored about the $x$ axis) for $G a=25$ and $E o=1$ (point $D$ ). This point is slightly closer

to the boundary separating regions $I$ and $I I$, i.e. with slightly higher inertia than the bubbles at point $A$, whose dynamics is investigated in Fig. 3(a). It can be seen in Fig. 14(a) that the bubbles ' 1 ' and ' 2 ' migrate away from 
(a)

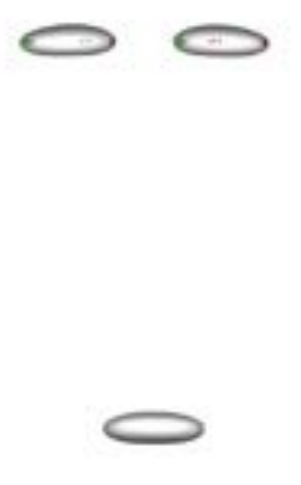

(d)

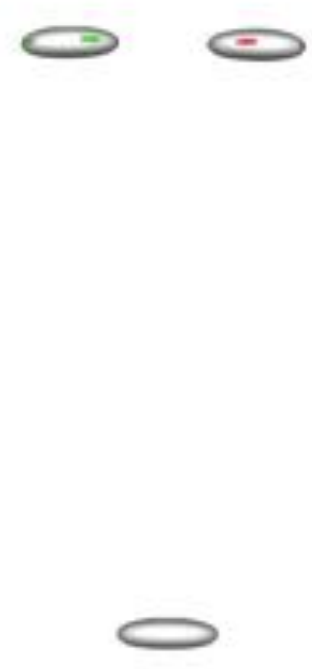

(b)

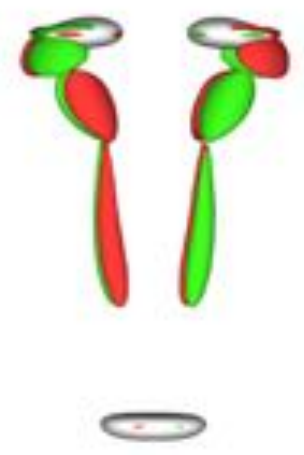

(e)

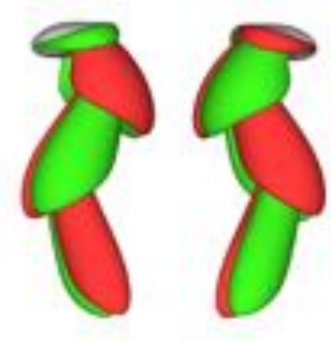

(c)

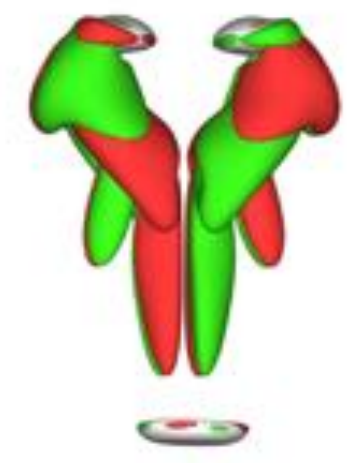

(f)

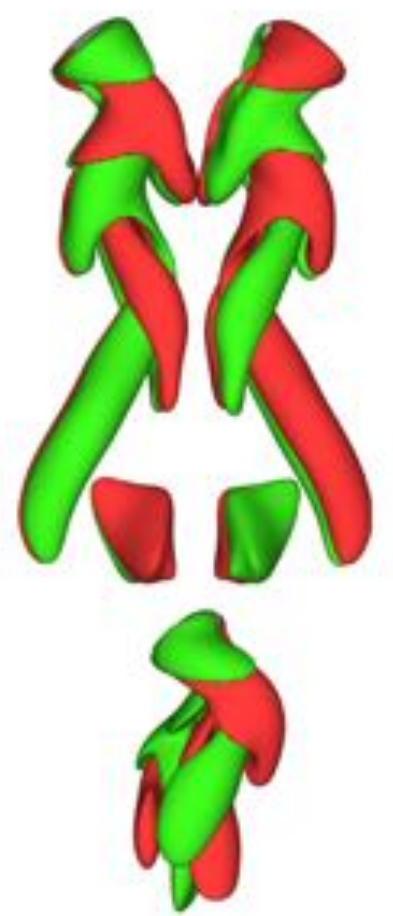

FIG. 10. Isosurfaces for $z$ vorticity at $t=15$ (a,b,c) and $t=30$ (d,e,f) for (a,d) $G a=22.4$, (b,e) $G a=32$ and (c,f) $G a=60$. The positive and negative values are shown by red and green colors respectively; $\omega_{z}= \pm 0.3$. The top and bottom panels in each subfigure are for two bubbles and single bubble, respectively. The rest of the parameter values are the same as in Fig. 3 .

each other with some path oscillations at the earlier times. The wavelength of these initial oscillations are larger than that of the bubbles at point $A$ (Fig. 3(a)). However, the higher surface tension (low Eo) suppresses asymmetrical shape deformations of the bubbles as compared to those associated with point $A$. This in turn minimises the path oscillations of the bubbles at later times. In Fig. 14(a), the bubbles are initially separated by a distance $q=3$ in the $y$ coordinate.

Then we investigate the rising dynamics of the bubbles at point $F$ for two values of $q$, i.e. $q=4$ and 2.2. The value of $E o$ for point $F$ is the same as that of point $A$, but $G a$ is slightly higher than that of point $A$. In Fig. 14(b) $(q=4$, point $\mathrm{F}$ ), it can be seen that both the bubbles move away for each other progressively, and this increasing separation is modulated by oscillations. The oscillations in the top panel of Fig. 14(b) is larger than those observed in Fig. 3(a). In Fig. 14(c) $(q=2.2$, point F), top panel shows that when we increase the initial distance between the bubbles, the lateral motion of the bubble decreases. This can be visualised by comparing the top panels of Fig. 14(b) and (c). 
(a)

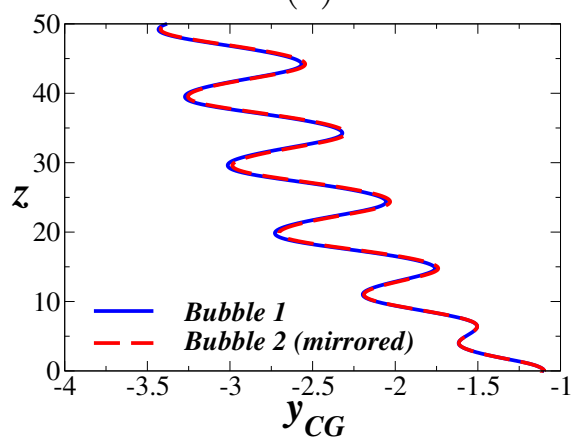

(d)

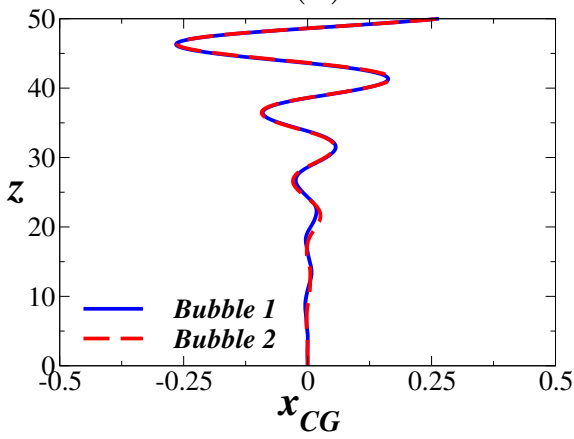

(b)

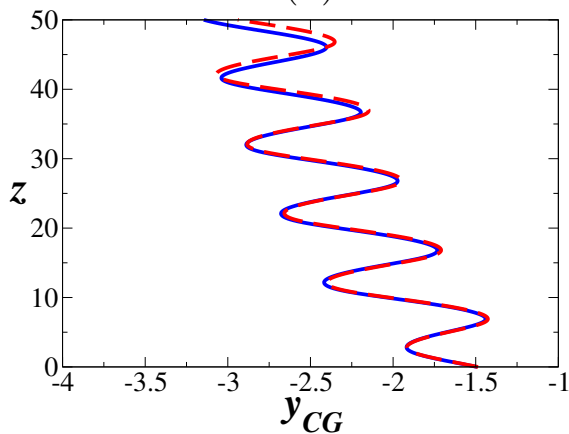

(e)

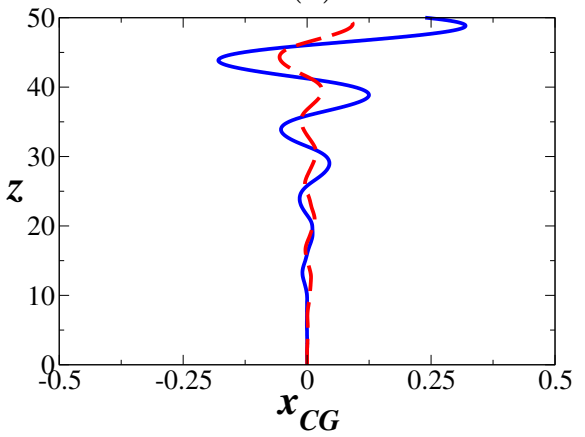

(c)

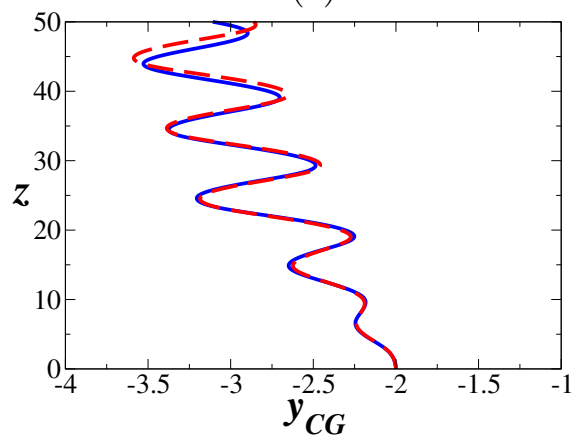

(f)

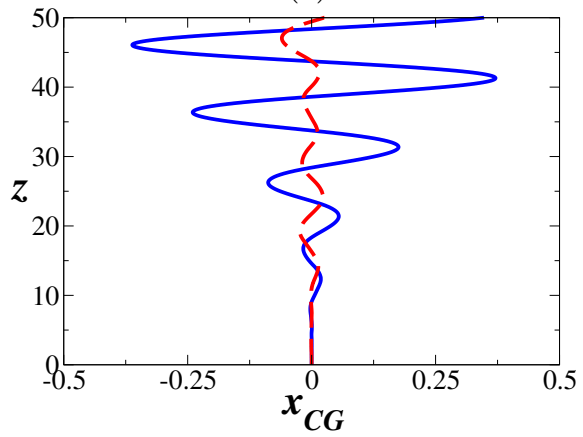

FIG. 11. Variation of $y_{C G}$ (top panels) and $x_{C G}$ (bottom panels) along the vertical path of the two bubbles released side by side for different values of $q$ : $(\mathrm{a}, \mathrm{d}) q=2.2$, (b,e) $q=3$ and $(\mathrm{c}, \mathrm{f}) q=4$. Here $G a=32$ and Eo= 4 (Point B). The position of the bubble initially placed at positive $y$ (bubble ' 2 ') has been mirrored about the $x$ axis in order to compare its path with that of the other bubble.

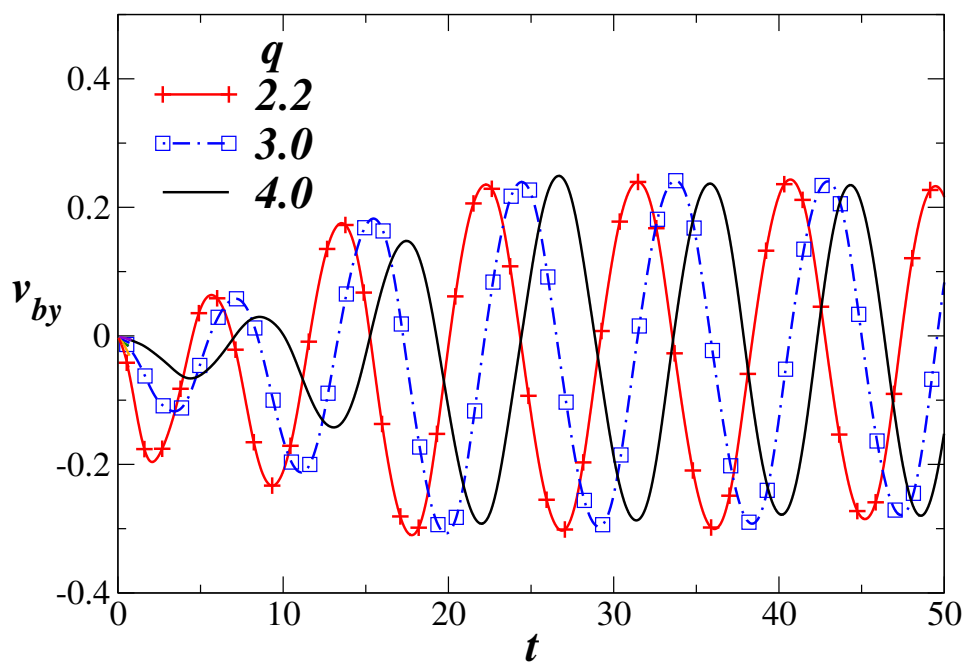

FIG. 12. Variation of $v_{b y}$ (velocity of the left bubble, bubble ' 1 ' in the $y$ direction) with time for different values of $q$. The rest of the parameter values are the same as in Fig. 11.

It can be seen in the bottom panels of Fig. 14(b) and (c) that their motion in the $x-z$ plane is negligible. Close inspection also reveals that the amplitude of oscillations increases progressively for $E o=4$ (see Fig. 14 (b) and (c)), while it decreases for $E o=1$ (see Fig. 14(a)). The increase in the amplitude of oscillations observed for $E o=4$ can be attributed to the larger deformation of the bubbles (due to lower surface tension) as compared to that for Eo $=1$.

Next, we investigate the dynamics at point $E$ in Fig. 14(d). This point is located in the central part of region III and corresponds to a set of parameters in the high inertial regime. It can be seen in the top panel of Fig. 14(d) that the motion of the bubbles in the $y-z$ plane is chaotic, although the paths of the two bubbles are perfect mirror 
(a)

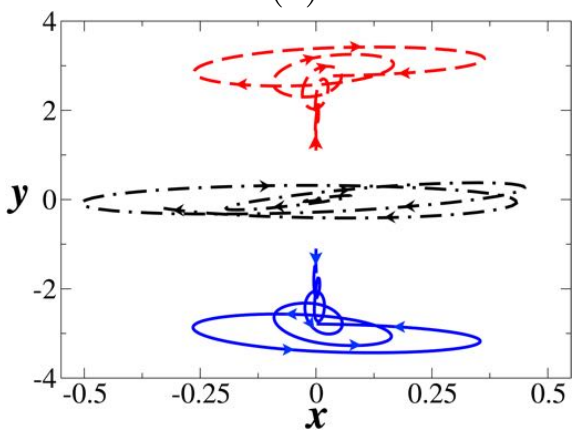

(b)

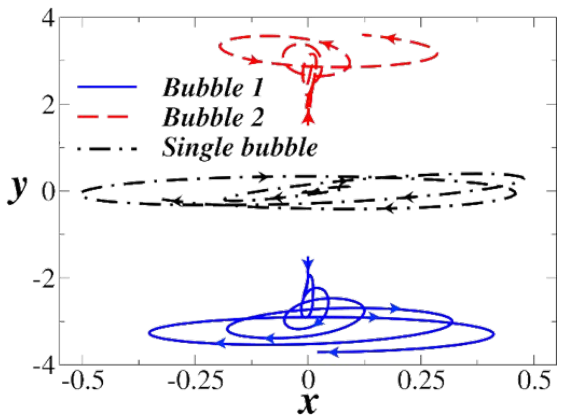

(c)

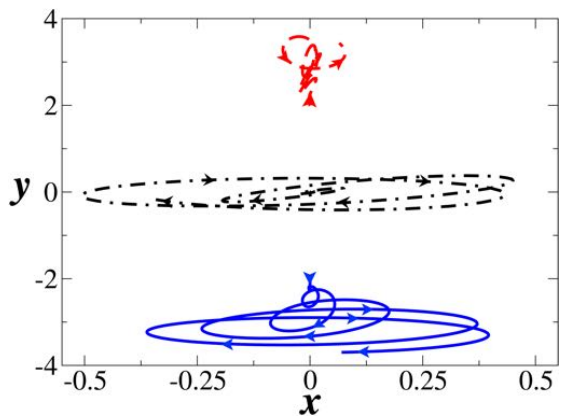

FIG. 13. The top view of the trajectories of the bubble for (a) $q=2.2$, (b) $q=3$ and (c) $q=4$. The top view of the trajectorie of the single bubble is also shown by black dot-dashed lines. The rest of the parameter values are the same as in Fig. 11.

images of each other about the $x$ axis till they reach upto $z \approx 40$, after that they deviate slightly from each other. The bottom panel of Fig. 14(d) presenting the motion of the bubbles in the $x-z$ plane shows that there is some foray into the third dimension as they rise.

(a)

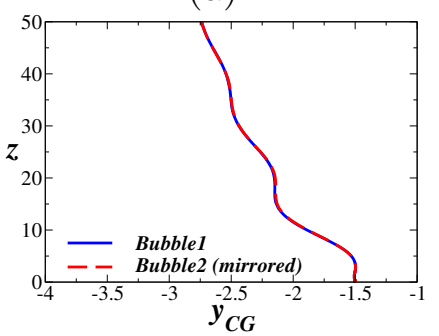

(e)

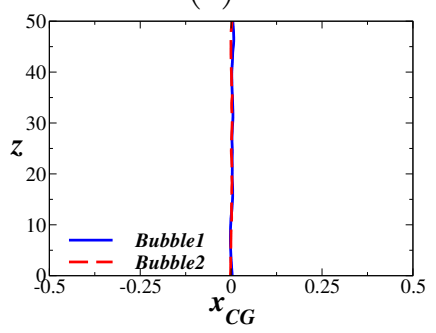

(b)

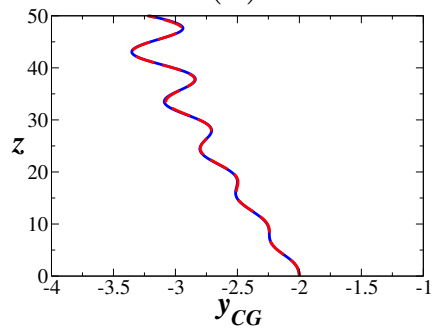

(f)

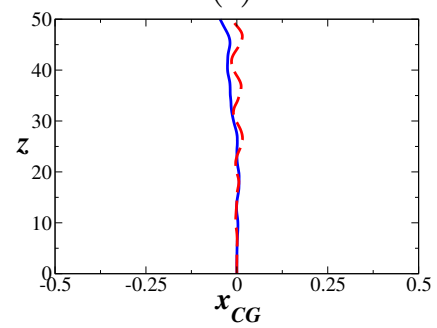

(c)

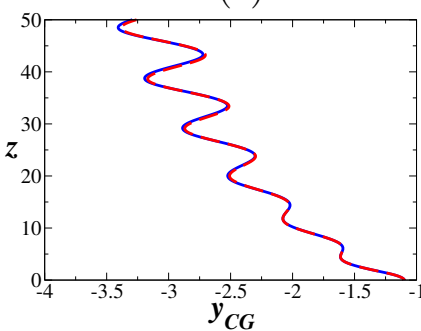

(g)

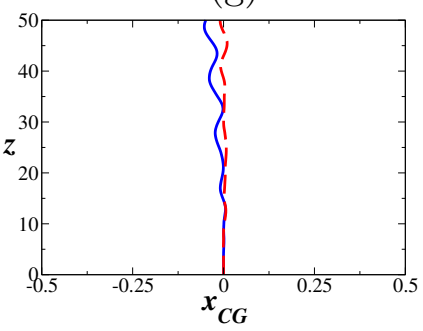

(d)

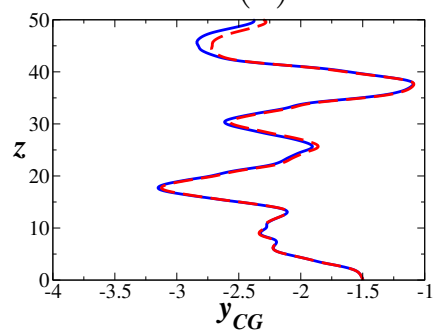

(h)

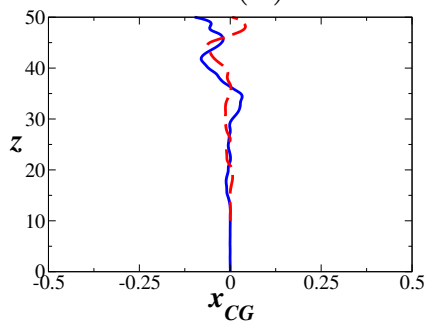

FIG. 14. Variation of $y_{C G}$ (top panels) and $x_{C G}$ (bottom panels) along the vertical path of the two bubbles released side by side: (a,e) $G a=25, E o=1, q=3$ (Point D), (b,f) $G a=25, E o=4, q=4$ (Point F), (c,g) $G a=25, E o=4, q=2.2$ (Point F) and (d,h) $G a=100, E o=2, q=3$ (Point E). The position of the bubble initially placed at positive $y$ (bubble '2') has been mirrored about the $x$ axis in order to compare its path with that of the other bubble.

\section{CONCLUDING REMARKS}

Two spherical objects rising through a heavier fluid in the Stokes flow regime would maintain their spacing. In particular, if the two objects are initially in the same horizontal plane, they would rise in straight vertical paths and the line separating them would be horizontal and of constant length. We ask how two identical inertial bubbles rising under gravity would respond to each other. We focus our attention on two regimes, termed regimes I and III in Tripathi et al. [10], in which a single bubble would display vertical rise in a straight line and oscillatory/zig-zag motion, respectively. We find that in both regimes, the vortices shed by the two bubbles interact strongly with each other, and the bubbles tend to move apart from each other as they rise. Further, the propensity to go to a spiralling or oscillatory state is increased by the presence of the second bubble. The interaction between the shed vortices organises the phase of the oscillations such that the motion of the bubbles tend to be mirror images of each other, 
except at significant inertia and high initial separation, where the motion is three dimensional and departures from mirror symmetry are observed particularly in the plane perpendicular to the separation vector. While the presence of the second bubble causes a drift along the separation line, it reduces the spiralling amplitude of each bubble. Increasing inertia has the effect of destabilising the steady trajectories, and chaotic motion is observed at high inertia, in a regime where a single bubble would display periodic oscillations. Interestingly however, the mirror symmetry is preserved in the chaotic regime as well, which speaks of a phase-locking in the vortex shedding of the two bubbles. A stability analysis of this flow will confirm our findings.

\section{ACKNOWLEDGMENTS}

The TIFR Centre for Interdisciplinary Sciences and the Indian Institute of Technology, Hyderabad are thanked for support. KS thanks the Indian National Science Academy for financial support. RG thanks the support by the Ministry of Earth Sciences, Government of India, under the Monsoon Mission Project on the Bay of Bengal. MT thanks the Department of Science and Technology for partially funding this research (Project number: DST/EES/2015037).

[1] W. D. Deckwer, Bubble column reactors (Wiley, 1992).

[2] B. Aboulhasanzadeh and G. Tryggvason, "Effect of bubble interactions on mass transfer in bubbly flow," Int. J. Heat Mass Transfer 79, 390-396 (2014).

[3] Y. Hallez and D. Legendre, "Interaction between two spherical bubbles rising in a viscous liquid," J. Fluid Mech. 673, 406-431 (2011).

[4] Duncan C Blanchard, "The electrification of the atmosphere by particles from bubbles in the sea," Prog. Oceanogr. 1, 73-202 (1963).

[5] Mark Sussman and Elbridge Gerry Puckett, "A coupled level set and volume-of-fluid method for computing 3D and axisymmetric incompressible two-phase flows," J. Comput. Phys 162, 301-337 (2000).

[6] W Dijkhuizen, M van Sint Annaland, and JAM Kuipers, "Numerical and experimental investigation of the lift force on single bubbles," Chem. Eng. Sci. 65, 1274-1287 (2010).

[7] MW Baltussen, JAM Kuipers, and NG Deen, "A critical comparison of surface tension models for the volume of fluid method," Chem. Eng. Sci. 109, 65-74 (2014).

[8] R. Clift, J. R. Grace, and M. E. Weber, Bubbles, Drops and Particles Academic (1978) p. 346.

[9] D. Bhaga and M. E. Weber, "Bubbles in viscous liquids: shapes, wakes and velocities," J. Fluid Mech. 105, 61-85 (1981).

[10] M. K. Tripathi, K. C. Sahu, and R. Govindarajan, "Dynamics of an initially spherical bubble rising in quiescent liquid," Nat. Commun. 6, 6268 (2015).

[11] G. Mougin and J. Magnaudet, "Path instability of a rising bubble," Phys. Rev. Lett. 88, 014502 (2002).

[12] A Tomiyama, GP Celata, S Hosokawa, and S Yoshida, "Terminal velocity of single bubbles in surface tension force dominant regime," Int. J. Multiphase Flow 28, 1497-1519 (2002).

[13] C. Veldhuis, Biesheuvel A., and L. van Wijngaarden, "Shape oscillations on bubbles rising in clean and in tap water," Phys. Fluids 20, 040705 (2008).

[14] Bernard Bunner and Grétar Tryggvason, "Direct numerical simulations of three-dimensional bubbly flows," Phys. Fluids 11, 1967-1969 (1999).

[15] T. Bonometti, J. Magnaudet, and P. Gardin, "On the dispersion of solid particles in a liquid agitated by a bubble swarm," Metall. Mater. Trans. B 38, 739-750 (2007).

[16] I. Chakraborty, G. Biswas, and P. S. Ghoshdastidar, "A coupled level-set and volume-of-fluid method for the buoyant rise of gas bubbles in liquids," Int. J. Heat Mass Transfer 58, 240-259 (2013).

[17] L. G. Leal, Laminar Flow and Convective Transport Processes (Butterworth and Heinemann, Stoneham, MA, 1992).

[18] J. B. W. Kok, "Dynamics of a pair of gas bubbles moving through liquid. part I. Theory." Eur. J. Mech. B/Fluids 12, 515-540 (1993).

[19] D. Legendre, J. Magnaudet, and G. Mougin, "Hydrodynamic interactions between two spherical bubbles rising side by side in a viscous liquid," J. Fluid Mech. 497, 133-166 (2003).

[20] J. B. W. Kok, "Dynamics of a pair of gas bubbles moving through liquid. part II. Experiment." Eur. J. Mech. B/Fluids 12, 541-560 (1993).

[21] R. H. Chen, W. X. Tian, G. H. Su, S. Z. Qiu, Y. Ishiwatari, and Y. I. Oka, "Numerical investigation on coalescence of bubble pairs rising in a stagnant liquid," Chem. Eng. Sci. 66, 5055-5063 (2011).

[22] P.C. Duineveld, "Bouncing and coalescence of bubble pair rising at high reynolds number in pure water or aqueous surfactant solutions," Appl. Sci. Res. 58, 409-439 (1998).

[23] T. Sanada, A. Sato, M. Shirota, and M. Watanable, "Motion and coalescence of a pair of bubbles rising side by side," Chem. Eng. Sci. 64, 2659-2671 (2009).

[24] Md. T. Islam, P. Ganesan, and J. Cheng, "A pair of bubbles rising dynamics in a xanthan gum solution: a CFD study," RSC Adv. 5, 7819-7831 (2015). 
[25] A. W. G. de Vries, A. Biesheuvel, and L. van Wijngaarden, "Notes on the path and wake of a gas bubble rising in pure water," Int. J. Multiphase Flow 28, 1823-1855 (2002).

[26] J. R. Velez-Cordero, P. Diego, S. amd Yue, J. J. Feng, and R. Zenita, "Hydrodynamic interaction between a pair of bubbles ascending in shear-thinning inelastic fluids," J. Non Newt. Fluid Mech. 166, 118-132 (2011).

[27] S. Shu and N. Yang, "Direct numerical simulation of bubble dynamics using phase-field model and Lattice Boltzmann Method," Ind. Eng. Chem. Res. 52, 11391-11403 (2013).

[28] Stéphane Popinet, "Gerris: a tree-based adaptive solver for the incompressible euler equations in complex geometries," J. Comput. Phys 190, 572-600 (2003).

[29] JU Brackbill, Douglas B Kothe, and C1 Zemach, "A continuum method for modeling surface tension," J. Comput. Phys 100, 335-354 (1992).

[30] Stéphane Popinet, "An accurate adaptive solver for surface-tension-driven interfacial flows," J. Comput. Phys 228, 58385866 (2009).

[31] JC Cano-Lozano, P Bohorquez, and C Mart inez-Bazán, "Wake instability of a fixed axisymmetric bubble of realistic shape," Int. J. Multiphase Flow 51, 11-21 (2013).

[32] José Carlos Cano-Lozano, Carlos Martinez-Bazan, Jacques Magnaudet, and Joël Tchoufag, "Paths and wakes of deformable nearly spheroidal rising bubbles close to the transition to path instability," Phys. Rev. Fluids 1, 053604 (2016). 


\section{APPENDIX: VALIDATION OF THE NUMERICAL SOLVER}

(a)
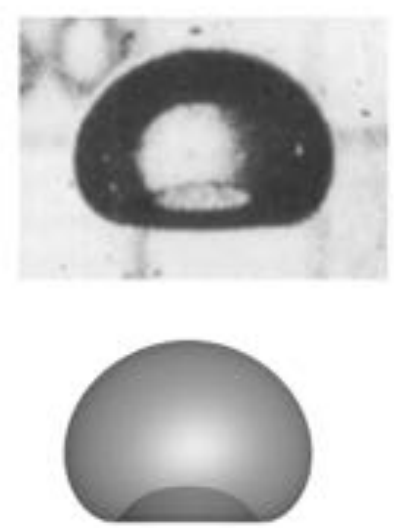

(b)
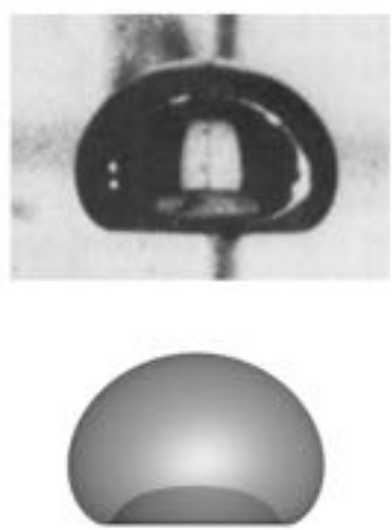

(c)
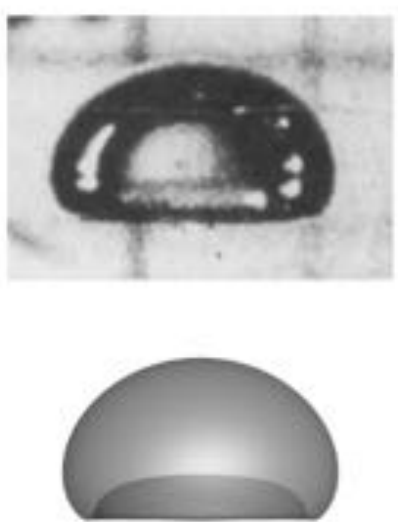

FIG. 15. Comparison of terminal shapes of an air bubble in water (large viscosity and density ratios) obtained from the present numerical solver with those of Bhaga \& Weber [9] for $E o=29$ and three values of $G a$ : (a) $G a=2.316$, (b) $G a=3.094$, and (c) $G a=4.935$. 